\title{
Influence of Size Effect on the Electronic and Elastic Properties of Graphane Nanoflakes: Quantum Chemical and Empirical Investigations
}

\author{
A. S. Kolesnikova, ${ }^{1}$ M. M. Slepchenkov, ${ }^{1}$ M. F. Lin, ${ }^{2}$ and O. E. Glukhova ${ }^{1}$ \\ ${ }^{1}$ Department of Physics, Saratov State University, Saratov 410012, Russia \\ ${ }^{2}$ Department of Physics, National Cheng Kung University, Tainan 701, Taiwan \\ Correspondence should be addressed to O. E. Glukhova; glukhovaoe@info.sgu.ru
}

Received 26 November 2014; Revised 23 April 2015; Accepted 23 April 2015

Academic Editor: Markus R. Wagner

Copyright (c) 2015 A. S. Kolesnikova et al. This is an open access article distributed under the Creative Commons Attribution License, which permits unrestricted use, distribution, and reproduction in any medium, provided the original work is properly cited.

\begin{abstract}
By application of empirical method it is found that graphene nanoflake (graphane) saturated by hydrogen is not elastic material. In this case, the modulus of the elastic compression of graphane depends on its size, allowing us to identify the linear parameters of graphane with maximum Young's modulus for this material. The electronic structure of graphane nanoflakes was calculated by means of the semiempirical tight-binding method. It is found that graphane nanoflakes can be characterized as dielectric. The energy gap of these particles decreases with increasing of the length tending to a certain value. At the same time, the ionization potential of graphane also decreases. A comparative analysis of the calculated values with the same parameters of single-walled nanotubes is performed.
\end{abstract}

\section{Introduction}

Graphane is a principal new and prospect material in nanoelectronics. Graphane was synthesized for the first time by the hydrogenation of graphene-a monolayer of graphite [1] . The crystal structure of graphane is two-dimensional and hexagonal. The hydrogen atoms are attached at opposite sides of the carbon atoms plane. It is necessary to distinguish the graphane nanoflakes and nanoribbons. The sizes of nanoflakes in the different directions differ no more than three times and do not exceed 100 nanometers.

The discovery of graphane has created prerequisites for the investigations of its properties and for the search of the probable application. For example, graphane has unique optical properties [2]. It is found that the dielectric functions of graphane nanoribbons spectra do not depend on the ribbon edge shapes and widths. Also, in the result of the graphane optical properties calculations, the presence of moderate anisotropy with respect to the type of light polarization is established. In [3], authors investigate thermal conduction in graphane nanoribbons by the nonequilibrium Green function method. It is demonstrated that thermal conduction can be effectively tuned by controlling the edge shape, width, and hydrogen vacancy concentration of graphane nanoribbons. Specifically, ballistic thermal conductance of graphane nanoribbons generally grows with the ribbon width. Big attention of researchers is devoted to investigation of the magnetic properties of graphane. In [4], it is shown that in the whole temperature range up to room temperature the hydrogenated graphene demonstrates a weak ferromagnetism. The origin of the magnetism is also determined to arise from the hydrogenated graphene itself.

Due to its unique properties, graphane has many applications. In particular, this material can find the application in the hydrogen energetics. It is established that the heating of graphane leads to release of the atomic hydrogen [5]. Therefore, graphane can be considered as one of the effective methods of hydrogen storage. Another significant application of graphane is its application in nanoelectronics as a base for the printed circuits with conducting and nonconducting sites [6]. 
One more possible field of graphane application is biosensing. In experimental work [7], the properties of graphane were considered for application in electrochemical oxidation of biomarkers. That can lead to finding of harmful ways for detection of biomarkers.

In present work [8], authors have studied theoretically the possibility of a superconducting state in two-dimensional hole-doped graphane. According to results of this work, the hole-doped graphane is a candidate for being a high temperature superconductor with a critical temperature as high as those of copper oxides.

One more perspective applied field of graphane is its use as thermoelectric materials for thermoelectric device. It is predicted that disordered armchair graphane nanoribbons with low thermal conductivity are promising candidates for constructing thermoelectric materials [9].

Although the properties of graphane nanoribbons have been studied extensively, the influence of size effect on the properties of graphane remained unexplored. At the same time, the variation of the geometrical dimensions of the objects could be an effective way to control the properties of graphane. The aim of this work is the theoretical investigation of the electronic and elastic properties of graphane nanoflakes by means of the quantum-chemical and empirical methods.

\section{Materials and Methods}

2.1. Empirical Method. To study the elastic properties and deformations of graphane nanoflakes we apply the empirical method based on the bond-order potential developed by Brenner [10]. The total energy is described by the sum of the binding energy $E_{b}$, the torsional energy $E_{\text {tors }}$, and the van der Waals energy $E_{\mathrm{vdW}}$ :

$$
E_{\text {tot }}=E_{b}+E_{\text {tors }}+E_{\mathrm{vdW}} .
$$

Each pair of covalently bonded atoms interacts via potential-energy:

$$
E_{b}=\frac{1}{2} \sum_{i=1}^{\mathrm{Nat}}\left(\sum_{j(\neq i)}\left(V_{R}\left(r_{i j}\right)-B_{i j} V_{A}\left(r_{i j}\right)\right)\right) .
$$

This is the binding energy. Here $V_{R}$ is the repulsive pair term, $V_{A}$ is the attractive pair term, and $r_{i j}$ is the distance between the chemically bonded atoms with numbers $i$ and $j$. The function $B_{i j}$ is a many-body term. This term was introduced to describe the specificity of the $\sigma-\pi$ interaction. So, the value of the binding energy depends on the position and chemical identity of atoms.

To describe nonbonded interaction between the atoms we have attended the torsional interaction energy to the total system energy. The torsional energy is given by the following formula:

$$
E_{\text {tors }}=\frac{1}{2} \sum_{i=1}^{\mathrm{Nat}}\left(\sum_{j \neq i}\left(\sum_{k \neq i, j}\left(\sum_{l \neq i, j, k} V_{\text {tors }}\left(\omega_{i j k l}\right)\right)\right)\right) .
$$

The torsional potential $V_{\text {tors }}\left(\omega_{i j k l}\right)$ is given as a function of dihedral angle $\omega$. The torsion angle $\omega_{i j k l}$ is defined in the usual way as the angle between the plane defined by the vectors $\mathbf{r}_{i k}$ and $\mathbf{r}_{i j}$ and that defined by $\mathbf{r}_{i j}$ and $\mathbf{r}_{j l}$. Here atoms $j$ and $k$ are not chemically bonded.

Van der Waals energy $V_{\mathrm{vdW}}$ defines the interaction between nonbonded atoms:

$$
E_{\mathrm{vdW}}=\frac{1}{2} \sum_{i=1}^{\mathrm{Nat}}\left(\sum_{j(\neq i)} V_{\mathrm{vdW}}\left(r_{i j}\right)\right) .
$$

Van der Waals interaction energy is described by the Morse potential as

$$
\begin{aligned}
V_{\text {Morse }}\left(r_{i j}\right)= & D_{e}\left(\left(1-\exp \left(-\beta\left(r_{i j}-r_{e}\right)\right)\right)^{2}-1\right)+E_{r} \\
& \cdot \exp \left(-\beta^{\prime} r_{i j}\right)
\end{aligned}
$$

where $D_{e}$ is the average bond energy, $E_{r}$ is the repulsion nucleus energy, and coefficients $\beta=10 \mathrm{~nm}^{-1}$ and $\beta^{\prime}=$ $40 \mathrm{~nm}^{-1}$. Calculated interatomic distance in graphite by described empirical method is equal to $0.1421 \mathrm{~nm}$.

2.2. Tight-Binding Method. Within this method the total energy is calculated by the following formula:

$$
E_{\text {tot }}=E_{\text {bond }}+E_{\text {rep }} \text {, }
$$

where $E_{\text {bond }}$ is the bond structure energy calculated as the sum of energies of the single-particle occupied states. $E_{\text {rep }}$ is the interelectron and internuclear repulsion term.

The bond structure energy is determined by the following formula:

$$
E_{\text {bond }}=2 \sum_{n} \varepsilon_{n}
$$

This expression is the sum of energies of the molecular orbitals obtained by diagonalizing the Hamiltonian, $n$ is the number of the occupied orbitals, and $\varepsilon_{n}$ is the energy of the single-particle orbitals.

The phenomenon energy can be expressed as a sum of two-body potentials:

$$
E_{\text {rep }}=\sum_{i<j} V_{\text {rep }}\left(\left|r_{i}-r_{j}\right|\right)
$$

where $i, j$ are the number of the interaction atoms; $r_{i}, r_{j}$ are the Cartesian coordinates.

The overlap matrix elements are calculated by the formulas which take into account four types of interaction, $s s \sigma, s p \sigma$, $p p \sigma$, and $p p \pi$, and the pair repulsive potential:

$$
\begin{aligned}
& V_{i j \alpha}(r) \\
& =V_{i j \alpha}^{0}\left(\frac{p_{3}}{r}\right)^{p_{1}} \exp \left\{p_{1}\left[-\left(\frac{r}{p_{2}}\right)^{p_{4}}+\left(\frac{p_{3}}{p_{2}}\right)^{p_{4}}\right]\right\}, \\
& V_{\text {rep }}(r) \\
& \quad=p_{5}\left(\frac{p_{3}}{r}\right)^{p_{6}} \exp \left\{p_{6}\left[-\left(\frac{r}{p_{2}}\right)^{p_{4}}+\left(\frac{p_{3}}{p_{2}}\right)^{p_{4}}\right]\right\},
\end{aligned}
$$


TABLE 1: Values of the parameters.

\begin{tabular}{lccccc}
\hline$\varepsilon_{s}, \mathrm{eV}$ & $\varepsilon_{p}, \mathrm{eV}$ & $V_{s s \sigma}^{0}, \mathrm{eV}$ & $V_{s p \sigma}^{0}, \mathrm{eV}$ & $V_{p p \sigma}^{0}, \mathrm{eV}$ & $V_{p p \pi}^{0}, \mathrm{eV}$ \\
\hline-10.93 & -5.991 & -4.344 & 3.969 & 5.457 & -1.938 \\
\hline$p_{1}$ & $p_{2}, \mathrm{~nm}$ & $p_{3}, \mathrm{~nm}$ & $p_{4}$ & $p_{5}, \mathrm{eV}$ & $p_{6}$ \\
\hline 2.796 & 0.232 & 0.154 & 22 & 10.92 & 4.455 \\
\hline
\end{tabular}

where $i$ and $j$ are the orbital moments of wave function and $\alpha$ presents the bond type $\left(\sigma\right.$ or $\pi$ ). The atomic terms $\varepsilon_{s}, \varepsilon_{p}$ are the atomic orbital energies of carbon which are located on the main diagonal of the Hamiltonian. The values of the $\varepsilon_{s}, \varepsilon_{p}$ atomic terms, the parameters $p_{n}(n=1,2,3,4,5,6)$, and the equilibrium overlap integrals $V_{i j}^{0}\left(V_{s s \sigma}^{0}, V_{s p \sigma}^{0}, V_{p p \sigma}^{0}\right.$, and $\left.V_{p p \pi}^{0}\right)$ are given in Table 1 . The coefficients $p_{1}, p_{4}$, and $p_{6}$ are the power exponents, the coefficients $p_{5}$ and $p_{3}$ are determined by the overall shape and steepness of the function, and the parameter $p_{2}$ is a cutoff distance for the hopping matrix elements and repulsive interactions.

The geometric and energy parameters for the carbon nanotubes and fullerenes obtained by the tight binding method are in good agreement with experimental data [11,12] and $\mathrm{ab}$ initio data [13]. The distances between carbon atoms and ionization potential are equal to $0.14 \mathrm{~nm}$ and $7.61 \mathrm{eV}$, respectively.

\section{Results and Discussion}

3.1. Elastic Properties of Graphane Nanoflakes. Elastic properties of graphane nanoflakes are investigated by means of the empirical method based on Brenner potential. We investigated graphane nanoflakes with lengths of 1-5 nm and width of $2.4 \mathrm{~nm}$. As a result of the optimization it is established that $\mathrm{C}-\mathrm{C}$ bond length is equal to $0.155 \mathrm{~nm}$, and $\mathrm{C}-\mathrm{H}$ bond length is equal to $0.112 \mathrm{~nm}$. Obtained results are in good agreement with the results of DFT PBE calculations (0.152 and $0.111 \mathrm{~nm}$ for C-C and C-H, resp.) [14].

In our study, graphane nanoflakes are subjected to axial compression load and stretching load. The edges of the structures were rigidly fixed. Reoptimization of the structures is carried out on each stage of numerical experiment of compression. We considered cases of compression for graphane nanoflakes on 1,2 , and $3 \%$. We observed a change of the graphane structure from planar to a wave-like at the $3 \%$ of compression (Figure 1). Thus, the behavior of graphane nanoflake at axial compression is similar to behavior of graphene under axial compression deformation [15]. The change of the deformation energy with axial compression is presented in Figure 2. From the figure it can be seen that the deformation energy increases quadratically with compression. However, a sharp energy decrease occurs at the $2.5 \%$ compression. One can assume that this fact indicates the phase transition from elastic deformation to plastic.

We calculated pseudo-Young's modulus for the investigated structure of graphane nanoflakes. Pseudo-Young's modulus is calculated on the base of the structure edge length. The deforming force acts on this edge. Pseudo-Young's modulus was chosen as a parameter of the graphane elasticity,

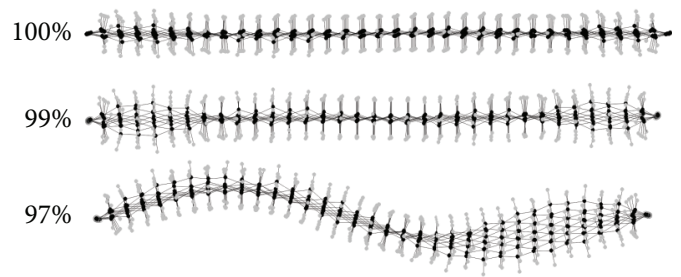

FIGURE 1: The change of graphane nanoflake structure under compression deformation.

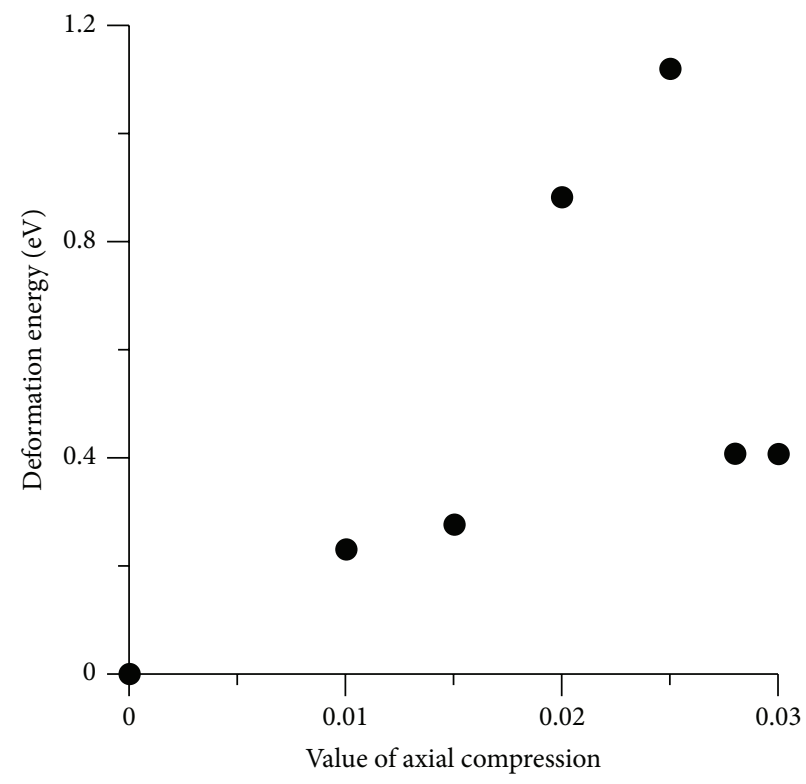

FIGURE 2: Dependence of the deformation energy on axial compression.

since the graphene is a nanoplate. Therefore, such estimation of the elasticity is more justified than classical Young's modulus. The algorithm for calculation of pseudo-Young's modulus is as follows.

(1) At the initial stage the optimization of the structure by means of the total energy minimization was performed.

(2) Then the optimized structure was compressed on $1 \%$. After that the edges of the structure were rigidly fixed, and a structure was optimized again.

(3) Pseudo-Young's modulus was calculated according to the formula

$$
Y_{p}=\frac{F}{L_{\text {ed }}} \frac{L}{\Delta L},
$$




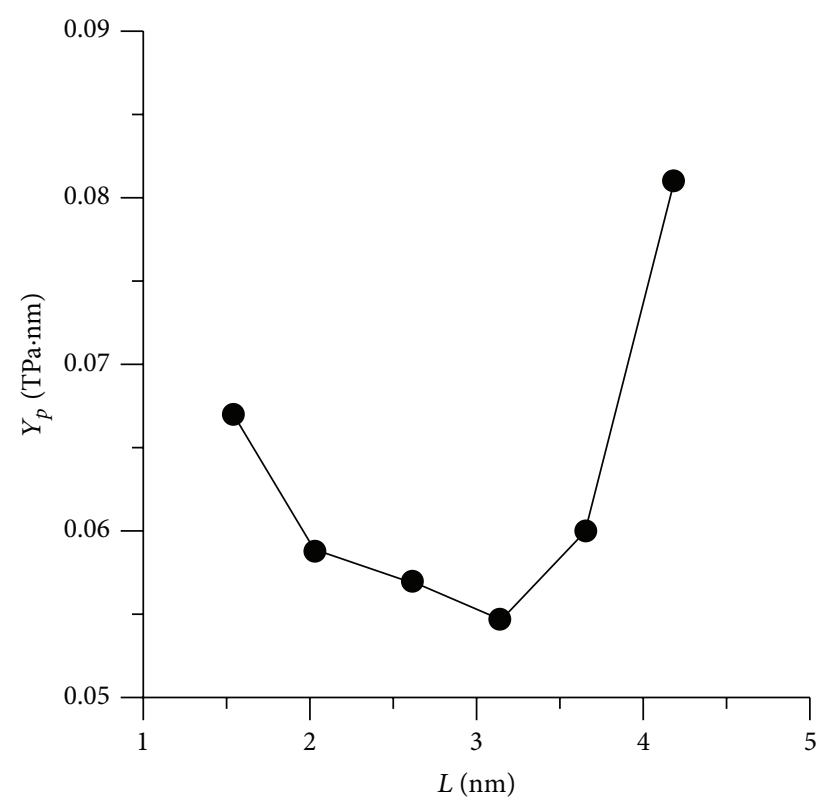

FIGURE 3: Dependence of the pseudo-Young's modulus on the graphane nanoflake length.

where $L$ is the length of graphane nanoflake; $L_{\text {ed }}$ is the edge length of graphane nanoflake; $F$ is the force, necessary for tube stretching. This force is determined according to the formula

$$
F=\frac{2 \Delta E}{\Delta L}
$$

where $\Delta E$ is energy of the elastic extension and $\Delta L$ is the length of the structure deformation.

Pseudo-Young's modulus was calculated according to the presented algorithm for graphane nanoflakes with length of $2.4 \mathrm{~nm}$ and width in the range of $1-5 \mathrm{~nm}$. The plot of the change for the elasticity parameter is shown in Figure 3. From the plot it can be seen that pseudo-Young's modulus does not change monotonically. It sharply increases starting with $3 \mathrm{~nm}$ length. This fact indicates an increase of elasticity. We can conclude that the elastic properties of the graphane nanoflakes are markedly inferior to the nanotubes on the elasticity. Pseudo-Young's modulus of the graphane nanoflakes reduced by $\sim 72 \%$ in comparison to graphene $(0.057-0.08$ $\mathrm{TPa} \cdot \mathrm{nm}$ of the graphane nanoflakes and $0.22-0.25 \mathrm{TPa} \cdot \mathrm{nm}$ [16] of zigzag and armchair nanotubes). Graphane nanoflakes show behavior that is typical for the case of plastic deformation.

3.2. Electronic Properties of Graphane Nanoflakes. Electronic properties of graphane nanoflakes were studied by a semiempirical tight-binding method. At first the atomic structure for graphane nanoflake of different sizes was optimized (by means of the total energy minimization by coordinates of the atoms). Then, the ionization potential and the energy gap of the spectrum were calculated by the electronic spectrum (the energy interval between the last occupied level and the first unoccupied level).

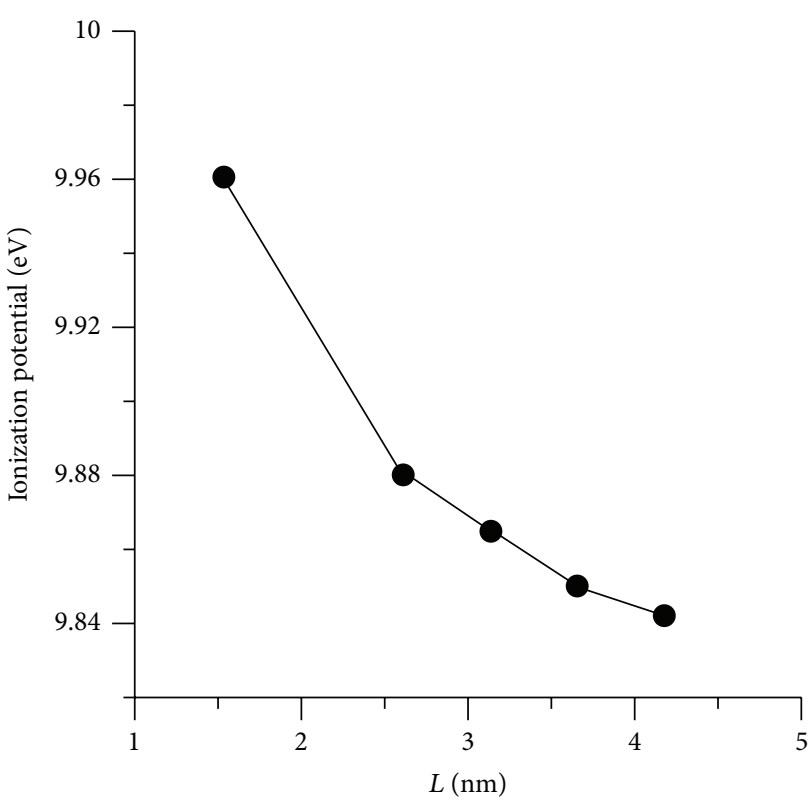

FIGURE 4: Dependence of the ionization potential on the graphane nanoflake length.

We carried out a set of the calculations for the ionization potential and the energy gap at the consecutive increase of the atomic skeleton for graphane particle. A plot of the ionization potential of graphane nanoflake on its length is presented in Figure 4. The linear decrease of the ionization potential with the increasing of the structure size is observed. It can be assumed that the ionization potential will tend to a work function of graphane with the further increase of the length.

The energy gap of the electron spectrum is also reduced with the increasing of the atomic skeleton of graphane particles. The plot of the energy gap of graphane nanoflakes on its length is presented in Figure 5. As can be seen from the plot, the energy gap takes on a value of about $7 \mathrm{eV}$. Therefore, we can conclude that graphane nanoflakes are good dielectrics.

The analysis of publications devoted to investigation of electron structures of hydrogenated graphene showed that electron properties of just periodic graphane nanostructures were studied. In our work appearance of size effect in electron and energetic features of graphane was proved for the first time. For periodic graphane structure of armchair-type it is established that the ionization potential is equal to $4.73 \mathrm{eV}$ and the energy gap of spectrum is equal to $6.05 \mathrm{eV}$ [17]. Analyzing the dependencies of the ionization potential and energy gap on graphane length presented in our paper, it can be concluded that the electron and energetic characteristics of graphane with growing of atomic skeleton will tend to values presented in [17].

\section{Conclusions}

The current paper presented results of the theoretical investigation of size effect influence on the elastic and electronic properties of graphane nanoflakes. Theoretical study of the deformation for graphane nanoflakes revealed that graphane 


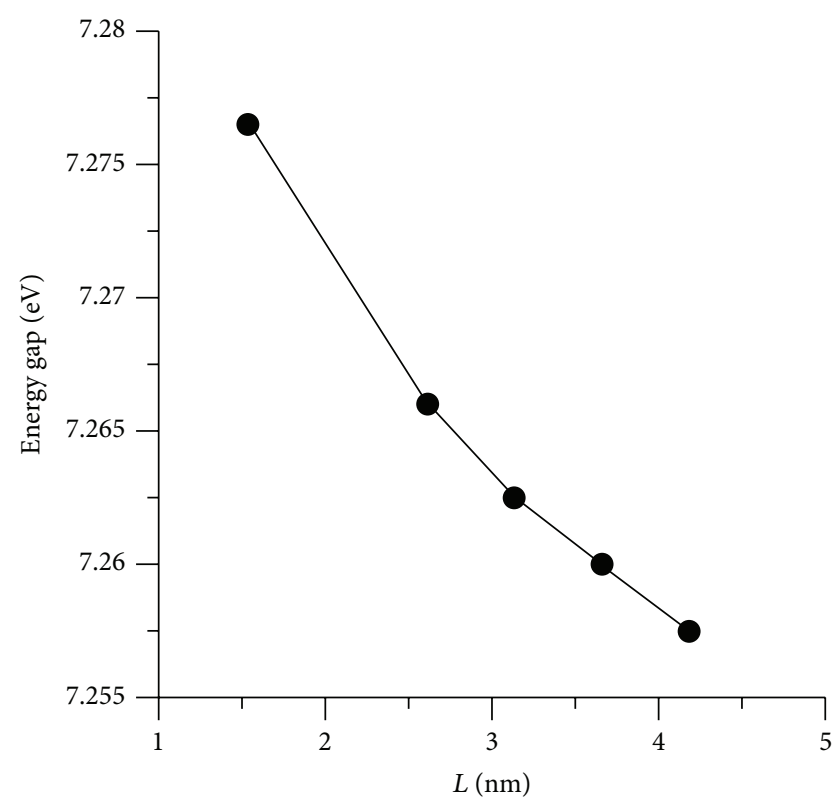

FIGURE 5: Dependence of the energy gap on the graphane nanoflake length.

nanoflakes are not elastic material. This is evidenced by small pseudo-Young's modulus as compared with value of nanotubes and the appearance of the plastic deformation of the structure at already $3 \%$ compression.

Also it is found that graphane nanoflakes are dielectric with sufficiently large energy gap of the electronic spectrum. Therefore, one can obtain contact tracks with electronic conductivity of desired configuration by removing of the hydrogen atoms from graphane surface. The remainder of the surface will remain dielectric.

\section{Conflict of Interests}

The authors declare that there is no conflict of interests regarding the publication of this paper.

\section{Acknowledgments}

The authors gratefully acknowledge funding of this work by Ministry of Education in the framework of the project of the state task (no. 3.1155.2014/K) and by Presidential scholarship 2013-2015 (Project no. SP-2302.2013.1).

\section{References}

[1] D. C. Elias, R. R. Nair, T. M. G. Mohiuddin et al., "Control of graphene's properties by reversible hydrogenation: evidence for graphane," Science, vol. 323, no. 5914, pp. 610-613, 2009.

[2] Y.-E. Yang, Y.-R. Yang, and X.-H. Yan, "Universal optical properties of graphane nanoribbons: a first-principles study," Physica E: Low-Dimensional Systems and Nanostructures, vol. 44, no. 7-8, pp. 1406-1409, 2012.

[3] D. Li, Y. Xu, X. Chen, B. Li, and W. Duan, "Tunable anisotropic thermal conduction in graphane nanoribbons," Applied Physics Letters, vol. 104, no. 14, Article ID 143108, 2014.
[4] A. Y. S. Eng, H. L. Poh, F. Šaněk et al., "Searching for magnetism in hydrogenated graphene: using highly hydrogenated graphene prepared via birch reduction of graphite oxides," ACS Nano, vol. 7, no. 7, pp. 5930-5939, 2013.

[5] O. G. Ziogos and L. Tsetseris, "Formation and properties of graphane superstructures," Journal of Physics: Condensed Matter, vol. 25, no. 8, Article ID 085301, 2013.

[6] M. Pashangpour, Z. Bagheri, and V. Ghaffari, "A comparison of electronic transport properties of graphene with hexagonal boron nitride substrate and graphane, a first principle study," European Physical Journal B, vol. 86, no. 6, article 269, 2013.

[7] Q. Peng, A. K. Dearden, J. Crean et al., "New materials graphyne, graphdiyne, graphone, and graphane: review of properties, synthesis, and application in nanotechnology," Nanotechnology, Science and Applications, vol. 7, no. 2, pp. 1-29, 2014.

[8] A. P. Durajski, "Influence of hole doping on the superconducting state in graphane," Superconductor Science and Technology, vol. 28, no. 3, Article ID 035002, 2015.

[9] H. Sahin, O. Leenaerts, S. K. Singh, and F. M. Peeters, "GraphAne: from synthesis to applications," http://arxiv.org/abs/ 1502.05804 .

[10] D. W. Brenner, "Empirical potential for hydrocarbons for use in simulating the chemical vapor deposition of diamond films," Physical Review B, vol. 42, no. 15, pp. 9458-9471, 1990.

[11] T. Yatsuhashi and N. Nakashima, "Direct ionization desorption of fullerene by intense femtosecond laser fields," The Review of Laser Engineering. Supplements, vol. 36, pp. 1000-1003, 2008.

[12] L. N. Sidorov, "Buckminsterfullerene, higher fullerenes, and their endohedral and fluorine derivatives," Physics of the Solid State, vol. 44, no. 3, pp. 413-418, 2002.

[13] L. Stobinski, J. Peszke, and H.-M. Lin, "Computational studies of SWCNTs capped by hemispheres of $\mathrm{C}_{60}$ fullerene, based on semi-empirical methods," Reviews on Advanced Materials Science, vol. 5, no. 4, pp. 363-370, 2003.

[14] J. O. Sofo, A. S. Chaudhari, and G. D. Barber, "Graphane: a twodimensional hydrocarbon," Physical Review B, vol. 75, Article ID 153401, pp. 1-4, 2007.

[15] O. Glukhova and M. Slepchenkov, "Influence of the curvature of deformed graphene nanoribbons on their electronic and adsorptive properties: theoretical investigation based on the analysis of the local stress field for an atomic grid," Nanoscale, vol. 4, no. 11, pp. 3335-3344, 2012.

[16] O. E. Glukhova and O. A. Terent'ev, "Theoretical study of the dependences of the young's and torsion moduli of thin singlelayer carbon zigzag and armchair nanotubes on the geometric parameters," Physics of the Solid State, vol. 48, no. 7, pp. 1411-1417, 2006.

[17] S. Lebègue, M. Klintenberg, O. Eriksson, and M. I. Katsnelson, "Accurate electronic band gap of pure and functionalized graphane from GW calculations," Physical Review B, vol. 79, Article ID 245117, 2009. 

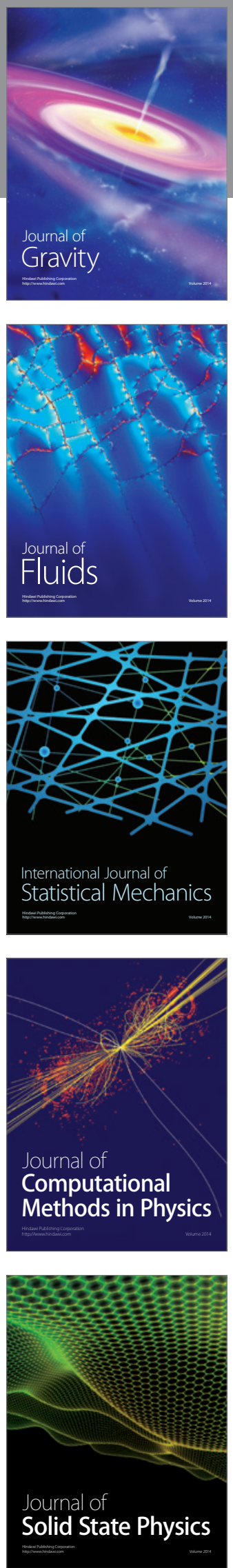

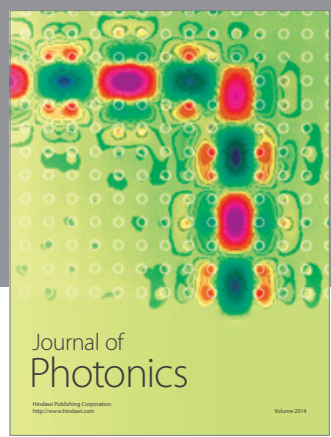

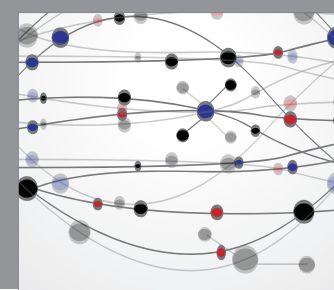

The Scientific World Journal

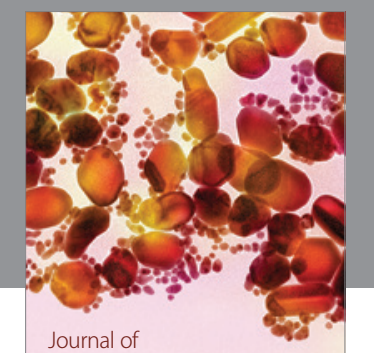

Soft Matter
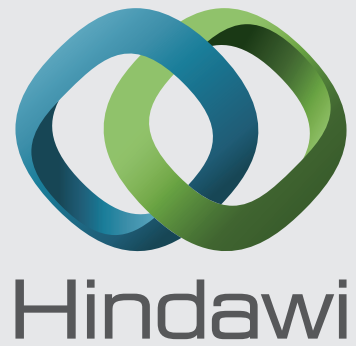

Submit your manuscripts at

http://www.hindawi.com
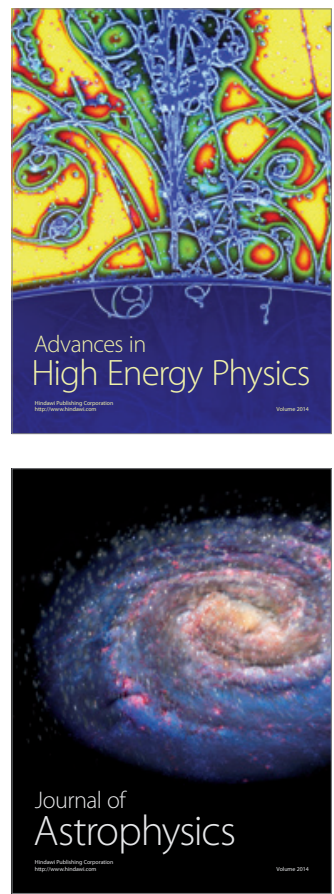
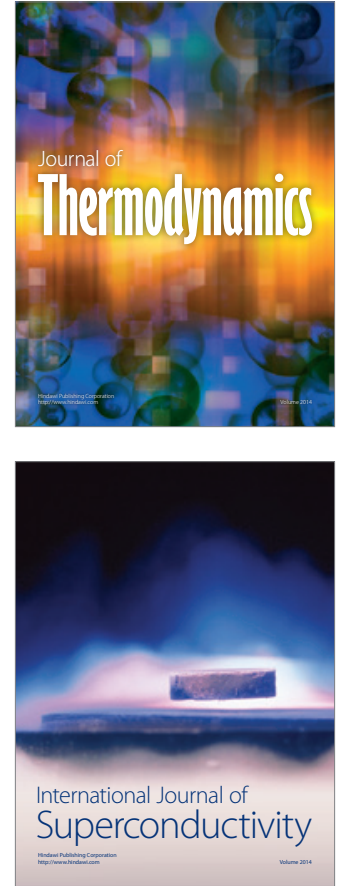
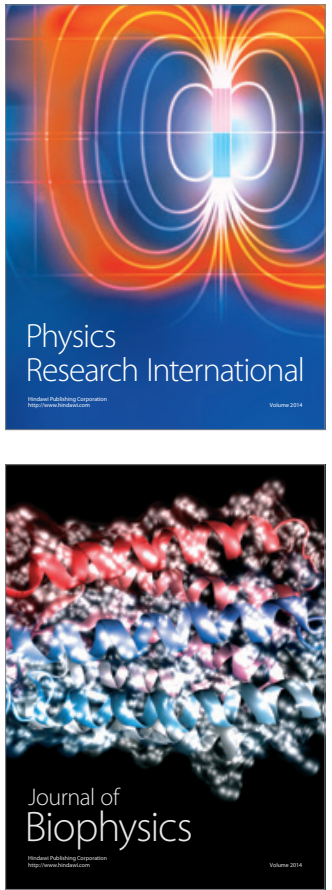
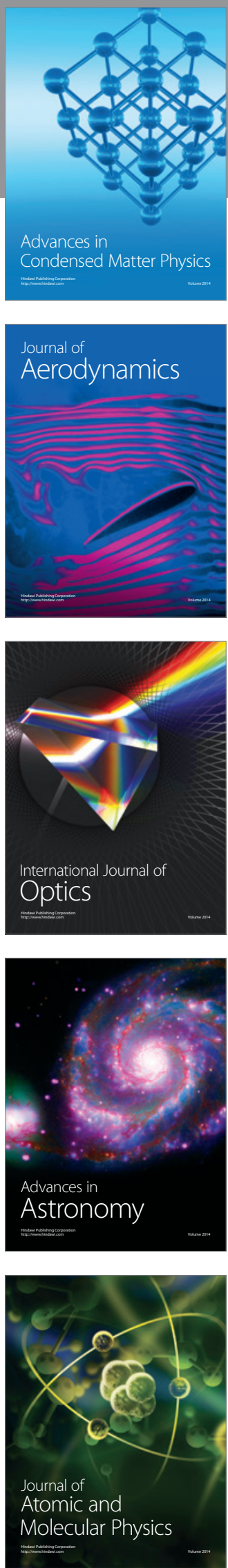